\title{
RADIOBIOLOGICAL INSTITUTE OF THE ORGANIZATION FOR HEALTH RESEARCH TNO, HOLLAND
}

$\mathrm{O}^{\mathrm{s}}$ $\mathrm{N}$ October 23, 1962, the new laboratory building of the Radiobiological Institute at Rijswijk, Holland, was formally opened by the Minister of Social Affairs and Public Health, Dr. G. M. J. Veldkamp. This ceremony marked the completion of the new facilities of the Institute, which was founded in 1956. Besides the main laboratory building (Fig. 1) there are three animal pavilions for the mouse colony, for an $S P F^{\prime}$-rat colony and for a monkey colony and a separate building housing the workshop and a conference room.

The Institute, which is under the direction of Dr. D. W. van Bekkum, has a broad research programme in the field of somatic radiation effects and their modification. Radiation damage to the living cell is investigated by means of biochemical and histological techniques, using not only tissues from animals exposed to radiation but also simpler systems such as mammalian cells cultured in vitro. For biochemical and biophysical investigation, cloning techniques as well as suspension cultures provide large quantities of cells. The reaction patterns of these cell populations in response to radiations of different ionization density give a clue to the fundamental processes involved in cellular radiation damage. In addition, these investigations are expected to provide a theoretical basis for a more rational X-ray therapy of tumours. In order to verify the patterns observed in vitro under various conditions in vivo, parallel investigations are carried out with transplantable tumours in experimental animals.
One of the functions of the Institute is to promote the clinical application of data obtained from experimental radiobiological research. Co-operation has been established with the Rotterdam Radiotherapeutical Institute and a radiologist is working under the joint auspices of the two Institutes on subjects of direct practical interest. A second major field of study is the prevention and treatment of radiation disease in which bone marrow transplantation in monkeys at present plays an important part. In addition, various immunological problems are being examined which are directly related to phenomena observed after transplantation of bone marrow. These more fundamental investigations are usually carried out with rats and mice. Clinical work on these problems is not performed at the Institute, but one of the staff members has a formal affiliation with the University Hospital at Leyden to organize and direct elinical studies in the field of bone marrow transplantation.

A third group of investigations concerns the toxic effects of radioactive isotopes. In recent years, emphasis has been laid primarily on the late effects of strontium-90 contamination in mice and the evaluation of various methods of treatment. The present new facilities permit an appreciable expansion of this work, both with respect to an increase in the number of experimental animals and the inclusion of other isotopes.

For some time the Radiobiological Institute has also carried out low-level determinations of radioactive isotopes in various biological products, but whenever these

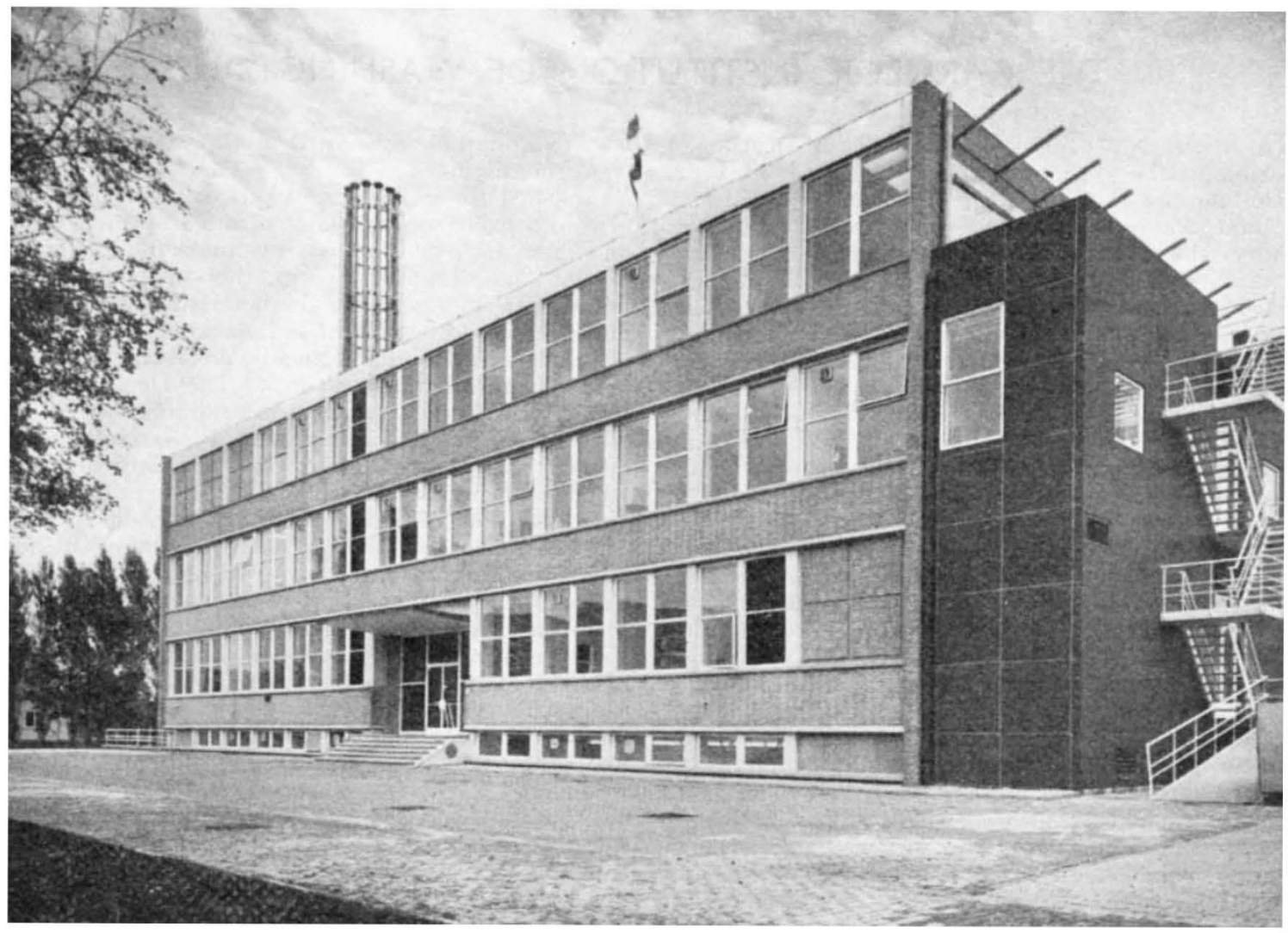

Fig. 1. Laboratory building of the Radiobiological Institute TNO, Rijswijk, Holland 
determinations have become a routine they have been taken over by other groups.

The Institute is well equipped with a variety of modern instruments and apparatus for biochemical and biophysical work. In addition, the radiobiological investigations require two special kinds of facilities, namely, radiation sources and experimental animals of very high quality. The Radiobiological Institute has at its disposal, besides small $\alpha$ - and $\beta$-sources, a 200-c. cæsium-137 $\gamma$-source for prolonged radiation of experimental animals and a 250-kV 'Maxitron' X-ray unit with circular beam used for short-term radiation. The special tube with which this apparatus is equipped makes it possible to irradiate a number of large animals simultaneously.

Lastly, a neutron generator is in use for measurements in the field of neutron dosimetry, and with this machine small biological objects such as cultured cells or small numbers of mice can be irradiated with neutrons. Cooperation has been established with the Reactor Centre of the Netherlands at Petten, which will make it possible to expose larger experimental animals to fission neutrons.

The laboratory has four specially designed rooms for biological work with higher levels of radioactive isotopes with installations for the removal of airborne and fluid waste.

In the Institute an attempt is made to maintain unusually high standards of health in the experimental animals used for radiobiological research. This policy, in which a great deal of effort and resources is involved, is followed for two reasons. In the first place, in many experiments the animals are exposed to total-body irradiation which greatly reduces their resistance to infection. When pathogenic micro-organisms are present even without causing manifest disease symptoms, there is a risk that the animals will develop the disease after irradiation. The consequent mortality will often prevent the successful conclusion of experiments. In the second place, a very prolonged observation period is required for the investigation of the so-called late effects of radiation and of isotope intoxication. These long-term experiments usually extend over the entire life-time of the animal. In the past, the health of the ordinary laboratory animals (conventional animals) often was so poor that most of them died of specific diseases at 'middle-age' or earlier, even without being exposed to other injurious factors.

The opinion held at the Radiobiological Institute is that long-term experiments with insufficiently selected animals will result only in loss of time and money. A start was therefore made some years ago, in co-operation with the Medical Biological Laboratory RVO-TNO, on a programme of far-reaching sanitation of the animal colonies. The procedure was based on Cæsarean-delivered, hand-fed young animals raised under strict isolation. The hand-feeding has now been abandoned since gnotobiotic animals are available for foster-nursing. The experience obtained so far has led to a modified approach -which is at present being pursued-namely, a multiplestep system for the production of satisfactory animals. These steps range from heavily protected gnotobiotic animals, via specific pathogen-free stocks to lightly protected production units. In cases of infection or other disorders in the production colonies, supplementation and, if necessary, total replacement can be made with animals from the next higher step. This project receives financial support from Euratom, and one of its objectives is to determine what grade of sanitation is optimal for the various kinds of radiobiological work.

The staff of the Institute includes, in addition to two physicists and two biochemists, seven medical graduates with training in different fields.

\section{M. van Putten}

\section{THE CARNEGIE INSTITUTION OF WASHINGTON}

\begin{abstract}
$Y^{\text {s }}$ EAR BOOK No. 61 * of the Carnegie Institution of Washington, covering the year ended June 30, 1962, and including the reports of the President, the Mount Wilson and Palomar Observatories, the Geophysical Laboratory, the Committee on Image Tubes for Microscopes, and the Departments of Terrestrial Magnetism, Plant Biology, Embryology, and of Genetics, marks the sixtieth anniversary of the Institution. The Institution's budget is now more than $\mathbf{2 . 8}$ million dollars, all of which is spent on six operating departments, whereas in 1905 the budget was less than 600,000 dollars, of which 302,000 dollars went to ten departments, about half of this being for the Solar Observatory on Mount Wilson, then under construction. Some 130,000 dollars were distributed in grants and nearly 30,000 dollars on subsidizing publications.

Experiments on maize had already begun, as had cytological research on Drosophila, and the Department of Genetics is still finding these lines of investigation productive. During the year, Dr. Barbara MeClintock confirmed the hypothesis that there is a high probability that genic control systems in maize and bacteria act in similar fashion. In the meantime, the group working on the application of cytology to genetics found that direct chromosomal breakage occurs in Tradescantia root tips in presence of 5-bromodeoxyuridine, which acts by modifying the base sequences in nucleic acid rather than the phosphate-sugar helixes attacked by deoxyribonuclease, and that Golgi bodies, one of the types of cytoplasmic organ-
\end{abstract}

* Carnegie Institution of Washington. Year Book, No. 61. July 1, 1961June 30,1962 . Pp. $x i+112+526+21$ plates. (Washington, D.C.: Carnegie Institution of Washington, 1962.) elle, exhibit different forms in the progression of microsporogenesis in Tradescantia. The Department has also established the molecular weight of the deoxyribonucleic acid of a bacteriophage known as $T 5$ by first establishing an ingenious pair of scales by analysing the deoxyribonucleic acid fragments of another phage (T2), and has found that the molecular characteristics of the deoxyribonucleic acid of phage lambda differ remarkably from those of other deoxyribonucleic acids, possessing a broad range of denaturation temperatures. The Department is now being terminated as a separate administrative unit and staff remaining in the Genetics Research Unit will cooperate with the new Laboratory of Quantitative Biology which is being organized.

In the Department of Plant Biology attention is still directed on the functions of chlorophyll and the accessory pigments in photosynthesis in which it has been interested since 1911. The persistence of the chemically unidentified material previously formed by illumination of chlorophyll $a$ has been measured, and by measuring oxygen exchange of Swiss chard chloroplasts four separate effects of light, each with its characteristic action spectrum, have been distinguished. Using the new technique of electron paramagnetic resonance, two distinctly different light-induced resonances have been observed, and it is concluded that the rapid-decaying signal, seen only when cells are illuminated, is associated with chlorophyll and arises from the 'primary' act of photosynthesis. Any inhibition of oxygen evolution, as by $3-\left(3^{\prime}: 4^{\prime}\right.$-dichlorophenyl)1 : 1-dimethyl urea, or by limiting the manganese-ion concentration in the growing medium, produces an enhanced rapid-decaying signal. Moreover, besides the 